\title{
sciendo
}

Int. J. of Applied Mechanics and Engineering, 2018, vol.23, No.4, pp.885-896

DOI: 10.2478/ijame-2018-0049

\section{NUMERICAL STUDIES OF NONLINEAR GEARING MODELS USING BOND GRAPH METHOD}

\author{
J. MARGIELEWICZ \\ Department of Logistics and Aviation Technologies \\ Silesian University of Technology, POLAND \\ J. WOJNAROWSKI \\ Higher Vocational School in Nowy Sącz, POLAND \\ S. ZAWIŚLAK* \\ Department of Computer Science and Automation \\ University of Bielsko-Biala, POLAND \\ E-mail: szawislak@ath.bielsko.pl
}

\begin{abstract}
The present paper is dedicated to computer simulations performed using a numerical model of a one-stage gear. The motion equations were derived utilizing the bond graph method. The formulated model takes into consideration the variable stiffness of toothings as well as an inter-tooth clearance which has been represented via discontinuous elements with so called dead zones. As a result of these assumptions, the nonlinear model was obtained which enables representation of the dynamic phenomena of the considered gear. In the paper, an influence of errors of gear wheels' co-operation on the character of excited dynamic phenomena was studied. The methodology of the analyses consists in utilization of the following tools: color maps of distribution of the maximal Lapunov coefficient and bifurcation diagrams. Based upon them, the parameters were determined, for which the Poincare portrait represents a structure of the chaotic attractor. For the identified attractors, the initial attractors were calculated numerically - which along with the changes of the control parameters are subjected to multiplication, stretching or rotation.
\end{abstract}

Key words: chaos, nonlinear vibrations, Lapunov exponent, bifurcations.

\section{Introduction}

Nonlinear systems have been intensively investigated experimentally and via versatile models especially since the second half of the 20th century. Qualitatively new phenomena were observed whose courses were evaluated as irregular due to new theoretical approaches. It is worth mentioning that nonlinear systems, especially these which behave in a chaotic manner, have been analyzed intensively and essentially since Edward Lorenz published in 1963, the results of computer simulations of a simplified model of fluid convection phenomena [Lorenz [1]]. In the aforementioned work, it was proved that a relatively slight change of initial conditions in a virtual area of the atmosphere, could be a cause of essentially high changes in some other area. Since the publication of Lorenz work, we can observe an intensive development of a new branch of science, i.e. the theory of chaos. Several papers have been published where the chaotic behavior has been shown for mechanical [Cai-Wan and Shiuh-Ming [2]; Khomeriki [3]; Awrejcewicz et al. [4], electric [Armand Eyebe Fouda et al. [5]] and robotized systems [Sajid et al. [6]]. Chaotic behavior of a dynamic system can be accurately observed when the modelled system is analyzed two times, changing slightly initial conditions of its work. In a general approach, the phenomena - whose courses are chaotic-occur when the analyzed systems

\footnotetext{
* To whom correspondence should be addressed
} 
have some special features, e.g.: irregular time histories of their characteristic variables as well as a continuous amplitude-frequency spectrum. Additionally, the Poincare cross-section has a complicated structure where a strange attractor can be observed [Dudkowski et al. [7]]. It is worth highlighting that an assessment of these phenomena can be performed utilizing several different numerical tools and approaches. Effective and useful numerical procedures are: the so called maximal Lapunov exponent [Awrejcewicz et al. [8]] and bifurcation diagrams showing splitting of the paths of solutions [Cai-Wan [9]].

In the present paper, the nonlinear model of the gear was analyzed via versatile numerical simulations. Free entering in meshing and outgoing from meshing of co-operating geared wheels are assured due to the tip and interteeth clearances. Interteeth clearance are one of the main sources of a nonlinear behavior of the system which is taken into account in the proposed mathematical model. Most frequently, it is modeled utilizing the special discontinuous function with the so called dead zone [Zhao Xin et al. [10]; Kokare and Patil [11]]. Due to simplification of numerical calculations, step-wise function characteristics of interteeth clearance is frequently approximated by means of, e.g. polynomial function [Saghafi and Farshidianfar [12]]. The step-wise function is not differentiable in some point, on the contrary, polynomial creates very precise substitute of class $C^{l}$. This method of modelling of interteeth clearance takes simultaneously into consideration the average stiffness of toothing, wherever an influence of one- and two-pair meshing is neglected. In consequence of such formulated assumptions, we obtain a model equivalent to Duffing's oscillator. The disordered phenomena which pass in gears in a chaotic manner, are mainly related to the excited forces due to the co-operation of a pair of gear teeth. Their values have an essential influence on the stiffness of toothing which, in turn, depends on the number of teeth being in mesh at particular time moment. From the theoretical point of view, the most simple method of identification of the stiffness of co-operating geared wheels - consists in considering the gear tooth as a restrained beam. Such simplification allows for an achievement of an approximate information, nevertheless it is sufficient for performance of quantitative and qualitative analyzes of the investigated system. More precise values of the toothing stiffness are determined by means of the formulas derived by Cai [13], Cai and Hayashi [14]. In the published results of investigations on particular models, the variable toothing stiffness was represented via the following different functions: harmonic [Wang et al. [15]], rectangular [Xiang et al. [16]] and trapezoid function [Litak and Friswell [17]].

Other factors influencing the gear dynamics are the so called transmission errors of the co-operating geared wheels [Łuczko 18]]. This parameter depends, among others, on preciseness of manufacturing and assembly of co-operating gear wheels. In the mathematical models, this variable is modelled by the harmonic function or the function being a superposition of several harmonic functions [Ghosh and Chakraborty [19]; Wang et al. [20]]. Additionally, numerical models of the gear sometimes take into account also the flexibility of shafts' bearings [Zhang et al. [21]]. The motion equations are usually derived based upon the classical methods of mechanics, which utilize the formalism of Lagrange's equations. However, in model-based investigations and simulation of gears, non-classical methods based on the graph theory are frequently utilized [Lang [22]; Wojnarowski et al. [23]; Drewniak and Zawiślak [24]]. Nevertheless, in the present paper, the bond graph method was applied for modelling [Lou and Tan [25]; Guo et al. [26]]. Bond graphs were defined in the second half of the 20th century by Paynter and are widely used nowadays. Their initial purpose was making it possible to analyze the stable and unstable states of electrical circuits. Later, Karnopp and Rosenberg extended the method, allowing for modelling of other types of technical systems, especially simultaneously in one common model which is a valuable feature of the bond graph approach. The most essential advantage of these graphs is an effective and handy modelling of complex dynamic systems consisting of subsystems of different technical nature. It is also worth mentioning that the basic convenience of the bond graph method consists in simulation of dynamics of technical systems, without explicit generation of mathematical notation of differential motion equations. The new approach consists also in consideration of clearances via a special summand in the equations.

\section{Formulation of the calculation model of the gear}

In the present paper, a one-stage cylindrical gear is the subject of its model investigations. Dynamic behavior of the gear is analyzed based on the model of a vibrating mechanical system of two degrees of 
freedom. The formulated phenomenological model (Fig.1) consists of two non-deformable discs of radiuses $R_{1}$ and $R_{2}$, respectively. Their inertial properties are represented by inertial mass moments $J_{l}$ and $J_{2}$. The discs rotate around the stiffly supported axes $O_{1}$ and $O_{2}$. The toothed wheels are coupled by means of two elements connected parallely, i.e. the elastic one - modelling stiffness of toothing $c_{Z}$ and the dissipative one modelling the energy losses of the co-working geared wheels $b_{Z}$. Additionally, in the formulated phenomenological model, an inter-meshing teeth clearance $L_{Z}$ and the so called transmission cooperation error (or just transmission error) of toothed wheels $e(t)$ is introduced. Motion of the gear is powered by an external drive moment $M_{N}$, additionally the gear is loaded by the loading moment $M_{O}$.

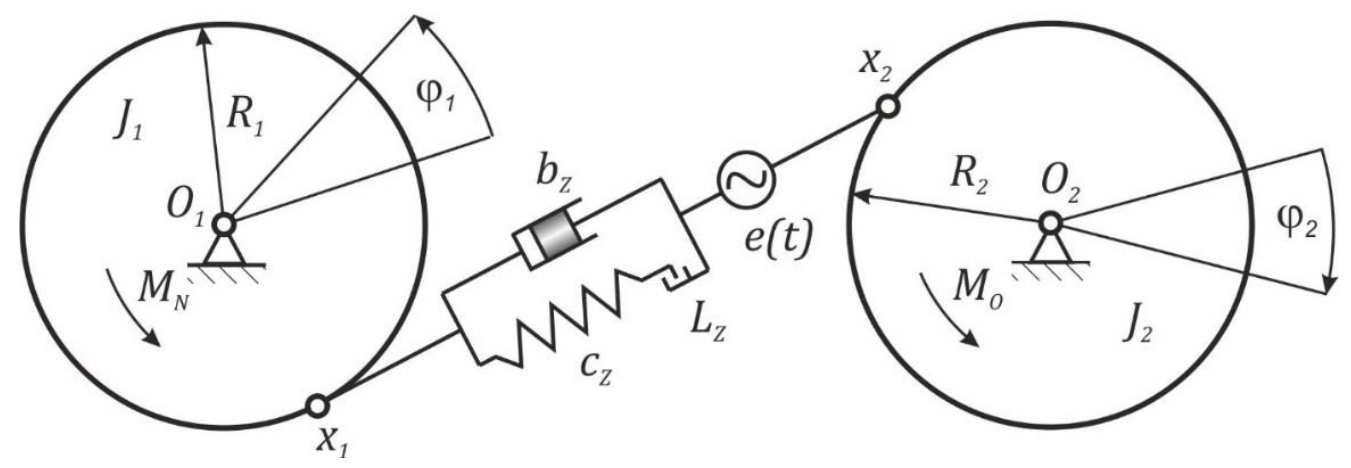

Fig.1. Phenomenological model of one-stage cylindrical gears.

Based on the phenomenological model of one-stage gear, its bond graph is built utilizing adequate rules (Fig.2). It represents the cause and effect relationships existing among the particular elements of the system.

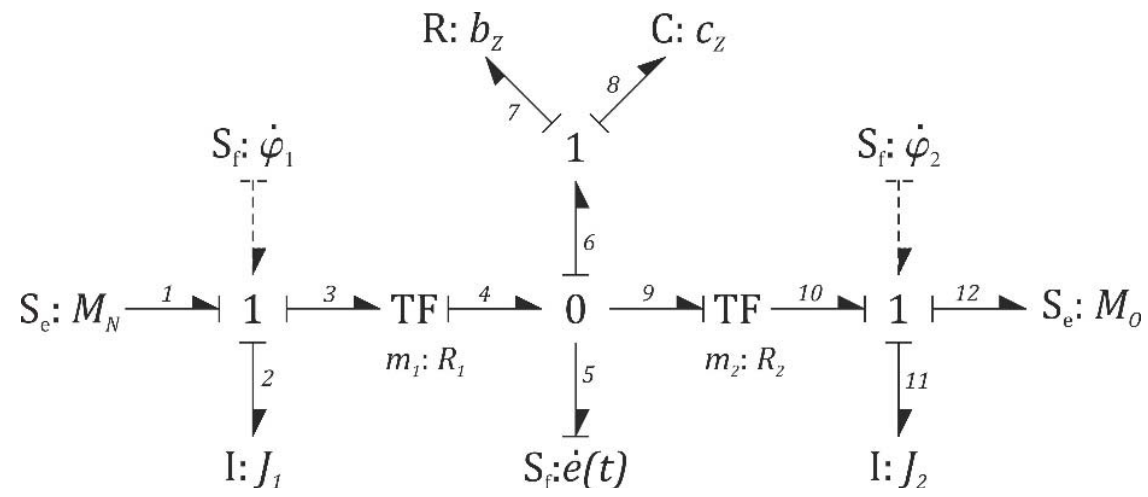

Fig.2. Bond graph representing Lagrange's phenomenological model of the gear.

The ideas and relations connected with the 0 -junctions and 1 -junctions of the bond graph constitute the formal basis for the derivation of differential motion equations. From the theoretical point of view, aiming at a quantitative description of the dynamics of the analyzed gear model, we do not need the explicit representation of motion equations. However, considering performance of qualitative investigations, in particular, aiming at a description of phenomena passing in a chaotic manner, it is necessary to know the mathematical model. In the scheme presented in Fig.2, a bond graph is shown in which two additional fiction edges of kinematic enforcement were distinguished by means of vertical dashed lines. Moreover, these edges are not to be described. Their goal is an assignment of flow variables to the 1-junctions, which represent angular deflections of the geared wheels. Due to consideration of the fictional edges of kinematical enforcements in the considered graph, we can apply differential causality of the elements accumulating the kinematical energy. The result of this approach consists in a possibility of using a simple procedure of derivation of differential motion equations in an explicit form. In general, it consists in writing down the relations existing between power variables for the 1-junctions incident to the fictional edges of kinematical enforcements 


$$
\left\{\begin{array} { c } 
{ e _ { 1 } = e _ { 2 } + e _ { 3 } , } \\
{ - e _ { 1 2 } = e _ { 1 1 } - e _ { 1 0 } , }
\end{array} \quad \left\{\begin{array}{c}
f_{1}=f_{2}=f_{3}=\dot{\varphi}_{1}, \\
f_{12}=f_{11}=f_{10}=\dot{\varphi}_{2} .
\end{array}\right.\right.
$$

The cause and effect relationships existing between the adequate variables of the bond graph, describing 0 -junction, 1 -junction and transformers are expressed by means of the following equations

$$
\left\{\begin{array}{c}
e_{6}=e_{7}+e_{8}, \\
f_{6}=f_{7}=f_{8},
\end{array},\left\{\begin{array}{c}
f_{4}=f_{6}+f_{9}+f_{5}, \\
e_{4}=e_{6}=e_{9},
\end{array},\left\{\begin{array} { c } 
{ f _ { 4 } = R _ { 1 } f _ { 3 } , } \\
{ e _ { 3 } = R _ { 1 } e _ { 4 } , }
\end{array} \quad \left\{\begin{array}{c}
f_{9}=R_{2} f_{10}, \\
e_{10}=R_{2} e_{9} .
\end{array}\right.\right.\right.\right.
$$

In case of elements accumulating kinematical and potential energy as well as elements which dissipate energy, the respectable mathematical relationships have the following form

$$
e_{2}=J_{1} \frac{d f_{2}}{d t}, \quad e_{11}=J_{2} \frac{d f_{11}}{d t}, \quad e_{7}=b_{Z} f_{7}, \quad e_{8}=c_{Z} \int f_{8} d t
$$

Aiming at derivation of motion equations in an explicit form, it is necessary to define the external loadings via the power $S_{e}$ and the flow $S_{f}$ sources, respectively

$$
e_{1}=M_{N}, \quad e_{12}=M_{O}, \quad f_{5}=\dot{e}(t) .
$$

After entering Eqs (2.2)-(2.4) into formula (2.1), the motion equations of the one-stage gear have the following form

$$
\begin{aligned}
& M_{N}=J_{1} \frac{d f_{2}}{d t}+R_{1} b_{Z}\left(R_{1} f_{3}-R_{2} f_{10}-\dot{e}(t)\right)+R_{1} c_{Z} \int\left(R_{1} f_{3}-R_{2} f_{10}-\dot{e}(t)\right) d t, \\
& -M_{O}=J_{2} \frac{d f_{11}}{d t}-R_{2} b_{Z}\left(R_{1} f_{3}-R_{2} f_{10}-\dot{e}(t)\right)-R_{2} c_{Z} \int\left(R_{1} f_{3}-R_{2} f_{10}-\dot{e}(t)\right) d t .
\end{aligned}
$$

Additionally, taking into account flow variables which are incident to the 1-junctions, one can rewrite the equation. Modification consists in introducing the description of the fictional edges being sources of flow variables. In consequence, the system of differential Eqs (2.5) turns into the final version

$$
\begin{aligned}
& M_{N}=J_{1} \ddot{\varphi}_{1}+R_{l} b_{Z}\left(R_{1} \dot{\varphi}_{1}-R_{2} \dot{\varphi}_{2}-\dot{e}(t)\right)+R_{1} c_{Z} \int\left(R_{1} \dot{\varphi}_{1}-R_{2} \dot{\varphi}_{2}-\dot{e}(t)\right) d t, \\
& -M_{O}=J_{2} \ddot{\varphi}_{2}-R_{2} b_{Z}\left(R_{1} \dot{\varphi}_{1}-R_{2} \dot{\varphi}_{2}-\dot{e}(t)\right)-R_{2} c_{Z} \int\left(R_{l} \dot{\varphi}_{1}-R_{2} \dot{\varphi}_{2}-\dot{e}(t)\right) d t .
\end{aligned}
$$

A qualitative evaluation of the forces generated during the meshing of the geared wheels, is easier to perform by means of the reduced 1-DOF model. The mentioned reduction is achieved via entering a new variable (using well known notation for the derivative of first and second order) $\dot{q}=R_{1} \dot{\varphi}_{1}-R_{2} \dot{\varphi}_{2}$

$$
m_{r e d} \ddot{q}+b_{Z}(\dot{q}-\dot{e}(t))+c_{Z} \int(\dot{q}-\dot{e}(t)) d t=m_{r e d} \delta
$$

where

$$
m_{\text {red }}=\frac{J_{1} J_{2}}{J_{2} R_{1}^{2}+J_{1} R_{2}^{2}} \quad \mu=\frac{M_{2}}{M_{1}}, \quad \delta=\left(\frac{R_{1}}{J_{1}}+\frac{R_{2} \mu}{J_{2}}\right) M_{1}, \quad e(t)=e_{1} \cos \left(\omega_{z} t\right) .
$$


Additionally, several other descriptions and relationships can be entered into the considered equations, i.e.: $\omega_{Z}=z_{l} \cdot \omega_{S}-$ mesh frequency, $\omega_{S}-$ motor angular velocity, $z_{l}$ - pinion teeth number. In consequence, the mathematical form of Eq.(2.7) can be simplified, entering the following replacement $\dot{u}=\dot{q}-\dot{e}(t)$. In consequence, Eq.(2.7), after some further substitutions has the final form

$$
m_{r e d} \ddot{u}+b_{Z} \dot{u}+c_{Z} u=m_{r e d} \delta+m_{r e d} \omega_{Z}^{2} e_{1}(t) \cos \left(\omega_{Z} t\right) .
$$

It should be underlined that till now the stiffness of meshing $c_{Z}$ has frequently been considered in a general manner, especially nonlinearities have been neglected. These nonlinearities result from interteeth clearances and fluctuating stiffness along the line of contact. During the numerical experiments, the fluctuating mesh stiffness was modelled by a periodic function

where

$$
c_{Z}(t)=c_{0}+c_{1} \cos \left(\omega_{z} t\right)
$$

$c_{0}$ - average mesh stiffness, $c_{1}-$ amplitude of the dynamic component.

Proper cooperation of the geared wheels is assured by an interteeth clearance, which is modelled (among other) by means of a discontinuous element with the so called dead zone. Adequate mathematical formulas for this element are as follows

$$
f\left(u, L_{Z}\right)=\left\{\begin{array}{cc}
u+0.5 L_{Z}, & u<-0.5 L_{Z}, \\
0, & -0.5 L_{Z} \leq u \leq 0.5 L_{Z}, \\
u-0.5 L_{Z}, & u>0.5 L_{Z} .
\end{array}\right.
$$

Taking into account relationships (2.9) and (2.10), the differential motion equations of the investigated gear model, after conversion into a dimensionless notation, can be written as follows

$$
\ddot{x}+h \dot{x}+\left[1+\alpha \cos \left(\omega_{G} \tau\right)\right] f(x)=f_{a v}+f_{e} \omega_{G}^{2} \cos \left(\omega_{G} \tau\right) .
$$

where

$$
\begin{aligned}
& \omega_{0}^{2}=\frac{c_{0}}{m_{\text {red }}}, \quad h=\frac{b_{Z}}{\sqrt{m_{r e d} c_{0}}}, \quad \alpha=\frac{c_{1}}{c_{0}}, \quad \omega_{G}=\frac{\omega_{z}}{\omega_{0}}, \\
& f_{a v}=\frac{m_{r e d} \delta}{L_{Z} c_{0}}, \quad f_{e}=\frac{e_{1}(t)}{L_{Z}}, \quad x=\frac{u}{L_{Z}} .
\end{aligned}
$$

As a consequence of the introduction of a dimensionless notation and a new co-ordinate $x$, which depends on dimensionless time $\tau=\omega_{0} t$, the dead zone is changed, which at present - in turn - is contained in the interval restricted by endpoints -1 and 1 . The formulated mathematical model constitutes the formal basis for of quantitative and qualitative computer simulations. The prepared model is more compound in comparison to those in literature, so we do expect that analyses would be more realistic and allow drawing more adequate conclusions.

\section{Investigation based on the model of the gear}

The model-based investigations were performed utilizing the numerical data gather in Tab.1, which represent the one-stage gear being a part of a lifting mechanism of the prototype bridge crane. During 
performance of computer simulations, inertias of the rotor of the electric motor $J_{S}$ and the drum of the hoist system $J_{B}$ were additionally taken into consideration. Moreover, these variables were recalculated for the pinion and the geared wheel, respectively. Additionally, in the simulations, it was assumed that the shafts connecting the gear with the motor and the cable drum are perfectly stiff.

Table 1. Parameters characteristic for the system under analysis.

\begin{tabular}{lcc}
\hline \multicolumn{1}{c}{ Name } & Description & Value \\
\hline Module & $\widehat{m}$ & $5[\mathrm{~mm}]$ \\
Tooth number of geared wheel 1 & $z_{1}$ & 14 \\
Tooth number of geared wheel 2 & $z_{2}$ & 85 \\
Mass moment of inertia of rotor & $J_{S}$ & $2.7\left[\mathrm{~kg} \mathrm{~m}^{2}\right]$ \\
Mass moment of inertia of drum & $J_{B}$ & $5.3\left[\mathrm{~kg} \mathrm{~m}^{2}\right]$ \\
Mass moment of inertia of wheel 1 & $J_{1}$ & $0.0011\left[\mathrm{~kg} \mathrm{~m}^{2}\right]$ \\
Mass moment of inertia of wheel 2 & $J_{2}$ & $1.12\left[\mathrm{~kg} \mathrm{~m}^{2}\right]$ \\
Average of toothing stiffness & $c_{0}$ & $5.03 \cdot 10^{8}\left[\mathrm{Nm}^{-1}\right]$ \\
Amplitude of dynamic component of mesh stiffness & $c_{1}$ & $3.27 \cdot 10^{7}\left[\mathrm{Nm}^{-1}\right]$ \\
Transmission error & $e_{1}(t)$ & $0.01[\mathrm{~mm}]$ \\
Nominal power of the drive motor & $P$ & $12[\mathrm{~kW}]$ \\
Nominal velocity of the drive motor & $n_{S}$ & $1450[\mathrm{rev} / \mathrm{min}]$ \\
\hline
\end{tabular}

The results of numerical calculations allow versatile analyses e.g.: an influence of the normalized frequency $\omega$. The transmission error of geared wheels $e_{1}$ on the gear dynamics is represented by means of a color map showing the distribution of the maximal Lapunov exponent $\lambda$ (Fig.3a), bifurcation diagrams (Figs 3b-3d), time responses, amplitude-frequency spectrums, Poincare's intersections (Fig.4) and the so called scale diagrams (Fig.5).

a)

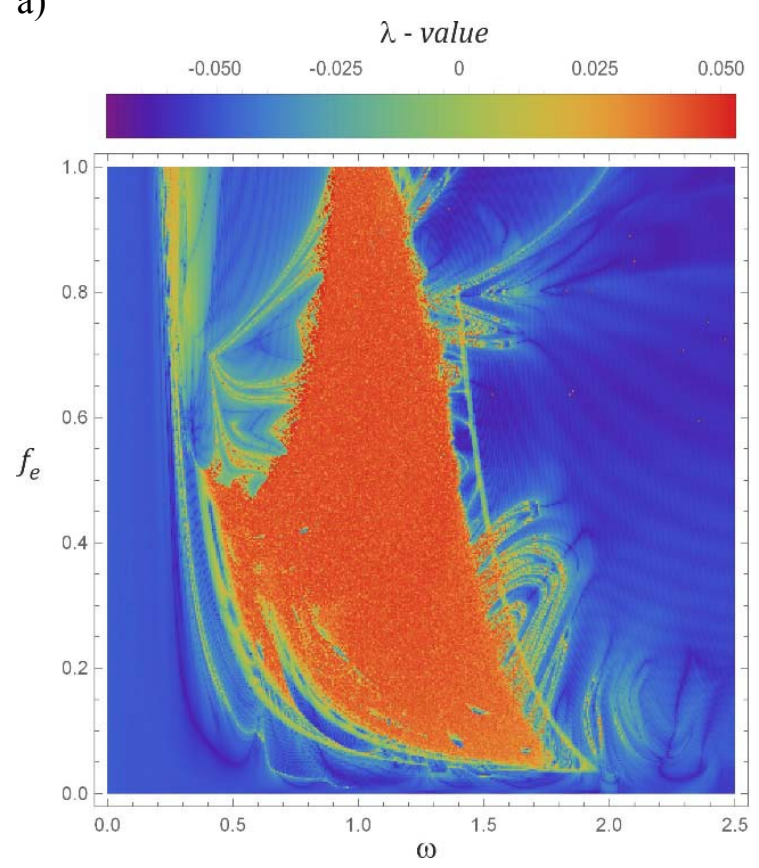

b)
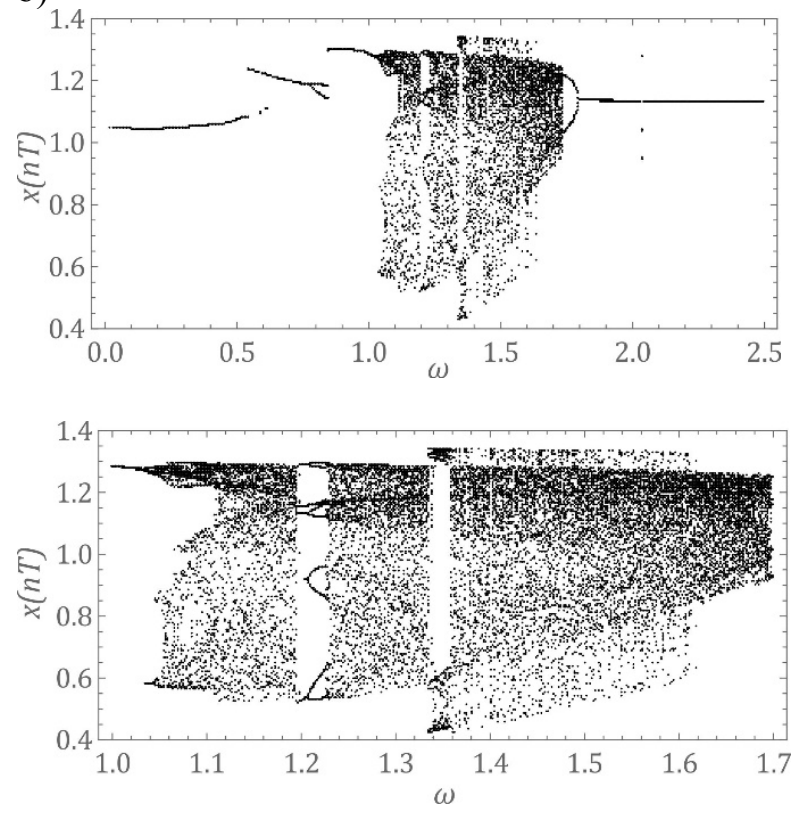

Fig.3. Results of numerical calculations: a) color map of distribution of the Lapunov exponent $h=0.12$, $\left.\alpha=0.065, f_{a v}=0.044, \mathrm{~b}\right)$ diagrams of bifurcations $f_{e}=0.1$, and their magnification. 
Moreover, other analyses were carried out to investigate the phenomena from different points of view, e.g.: an influence of the distribution of components of amplitude-frequency spectrum of normalized frequency $\omega_{G}$ on the structure of Poincare's cross sections. However, Poincare's cross sections were drawn on the background of phase image. For comparison, the corresponding numerical solutions received based on the mathematical model of the gear were presented - respectively - for time and frequency domains.
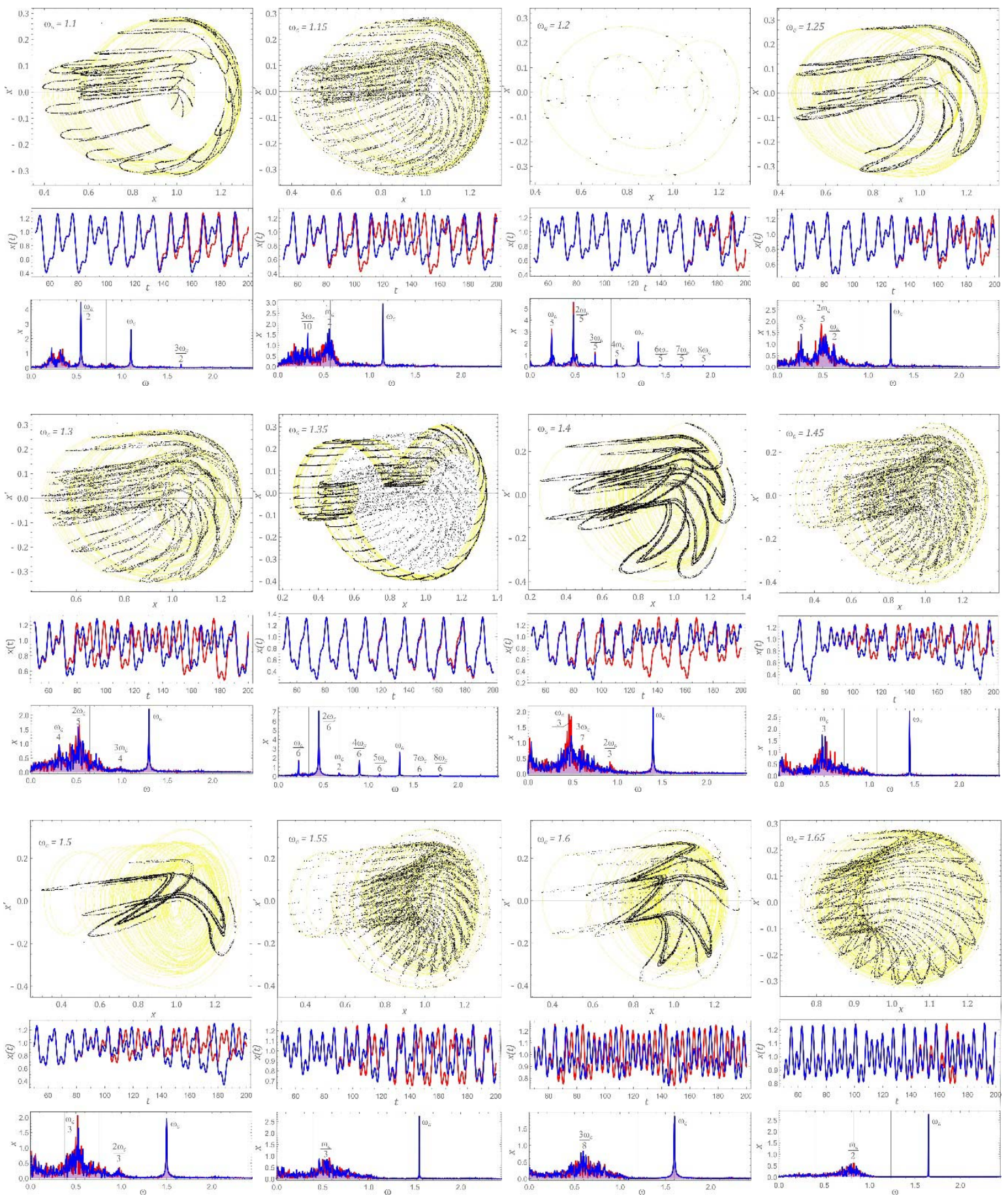

Fig.4. Influence of $\omega$ frequency and excited harmonic components on structure of Poincare's intersection. 
Aiming at detecting, which harmonic components are dominating in particular time moments - the so called scale diagrams were made which were obtained based upon the wavelet analysis.
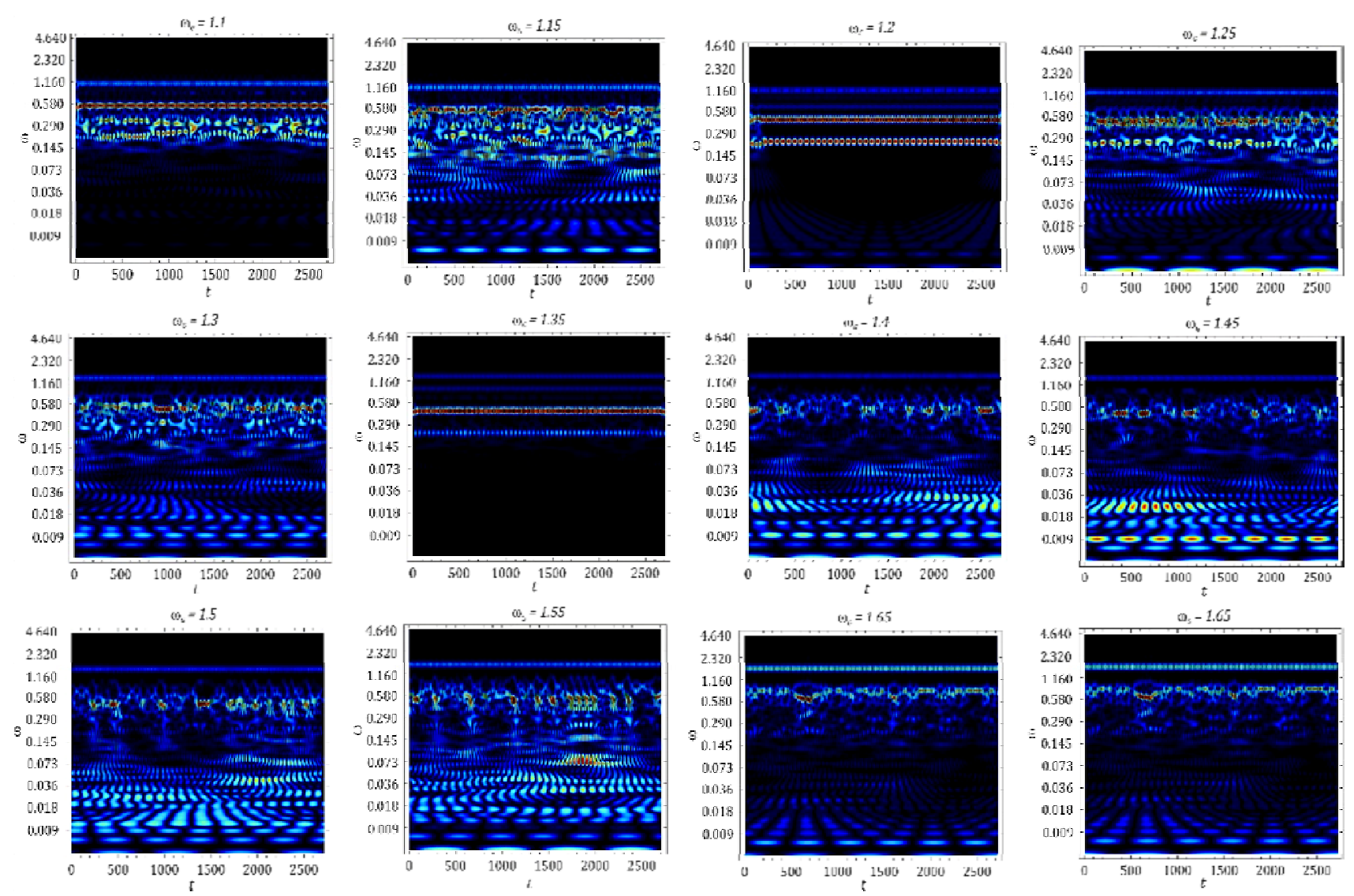

Fig.5. Color scale diagrams corresponding to Poincare's cross sections, which were generated for the chosen frequencies $\omega_{G}$.

Taking into consideration the similarity of Fourier spectrums, the numerical calculations were performed, aiming at detecting of the initial attractor for Poincare's cross section whose position is defined via the $l_{m}$. indicator. The images are registered in a special manner, i.e. every $l_{m}$. cycle of their course. Due to the fact that the position of the reference plane of Poincare's cross-section does not depend on the frequency of the source of dynamical excitations, the generated geometrical images of adequate cross-sections are equivalent to the enlarged primary Poincare cross-section.

Graphical representations of the initial attractors are presented on the background of Poincare's cross sections calculated for the control surface deflected at angle $2 \pi$. 

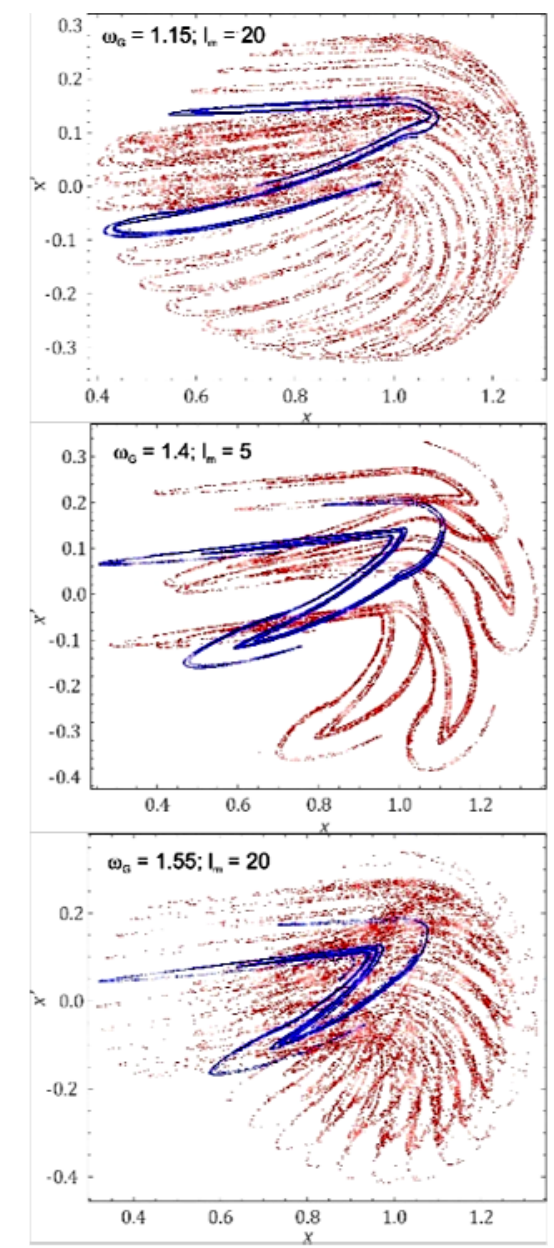
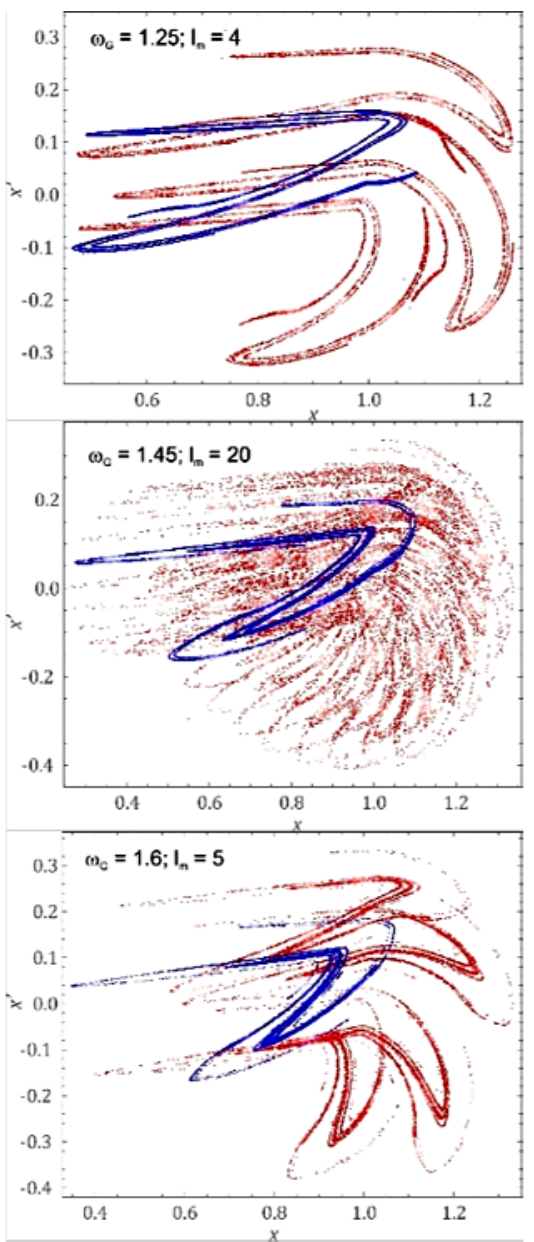
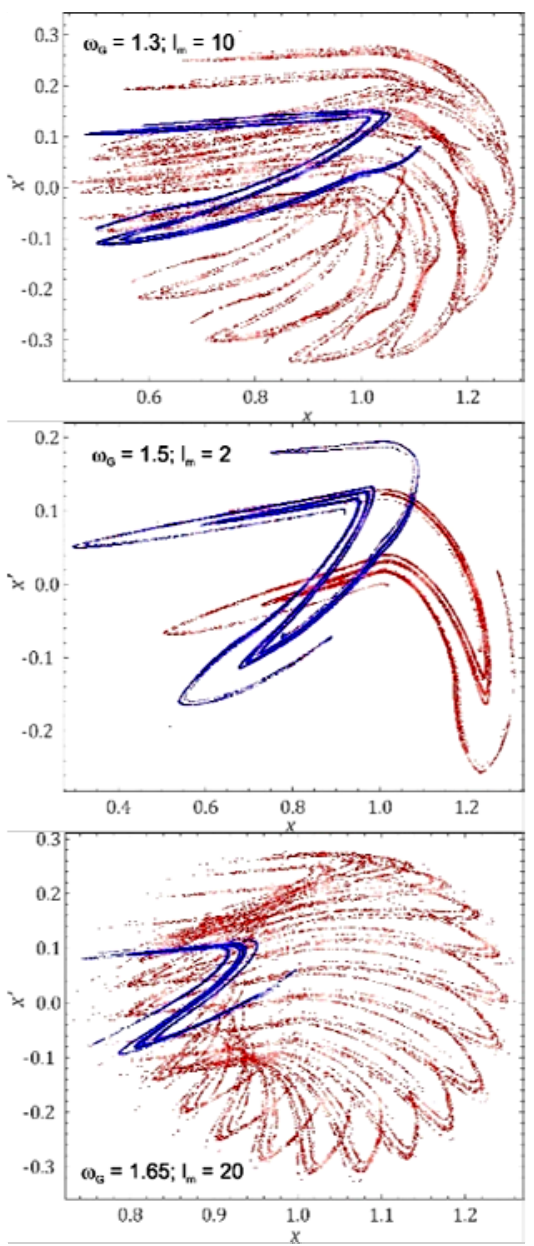

Fig.6. Initial Poincare's cross sections for the analyzed gear model.

\section{Analysis of results and final conclusions}

The color maps of the distribution of the Lapunov exponent inform the scientists in which areas of variability of control parameters the behavior of the gear can have a chaotic character. Moreover, the sensitivity of numerical solutions on the initial conditions was analyzed. These investigations consist in comparing the Fourier spectrums and time responses of generalized co-ordinates based on the mathematical model assumed for system modelling. This sensivity was evaluated for two slightly different initial conditions where the difference is equal to $\varepsilon=0.0001$. The methodology was briefly discussed in the introduction. Moreover, it is worth mentioning that generation of the Fourier spectrums showing the satisfied resolution implies utilization of the essentially longer time series then these used for obtaining the charts showing the time response of the considered system. The color map of the distribution of the maximal Lapunov exponent (Fig.3a) illustrates the chaotic behavior of the system within the variability range of the control parameter $\omega$ from approx. 0.35 up to approx. 1.7. Moreover, the range also changes with an increase of the transmission error of cooperating geared wheels $f_{e}$. In the present paper, the scope of investigations was restricted to an analysis of the dynamics of the gear, making the assumption that the transmission error is constant and equal to $f_{e}=0.1$. For the assumed value of parameter $f_{e}$, related to the particular cross section of the color map of the distribution of maximal Lapunov exponent, the diagrams of bifurcations were prepared (Fig.3b). Based on these diagrams, it was stated that in the considered gear, the chaotic phenomena dominate when the frequency of meshing is higher than the resonance frequency of the gear. In the performed 
investigations, the ratio of meshing frequency and the resonance frequency $\omega_{G}$ were equal to approx. 1.092. Moreover, they correspond to the situation, where some simplification of the model were incorporated, i.e. for a geared wheel having the moment of inertia $J_{l}$, the inertia of the rotor of the power motor was incorporated. Similarly, the inertia mass moment of the drum was incorporated in the second geared wheel moment of inertia $J_{2}$. This value was assumed as a limit and it has been changing by 0.05 , for particular cases - Poincare's cross sections, time responses and Fourier spectrums (Fig.4). It can be stated that the parameter $\omega_{G}$ had a decisive influence on the complexity of a structure of the attractor - which was presented on adequate Poincare's cross sections. In a general approach, it is possible to formulate an opinion that the attractors have usually the form of "small tongues of flames", whose number changes due to changes of frequency $\omega_{G}$. The obtained results of investigations related to domain of time and frequency proved that the system shows sensivity due to changes of the initial conditions for the established ratio $\omega_{G}$. Moreover, it is commonly known that the classical Fourier spectrums are useful investigation tools, delivering practical information related to the excited harmonic components of vibrations. However, based upon these data, it is impossible to determine the dominant frequencies in chosen time moments. Information about the excited harmonic components, corresponding to the particular time moments, is submitted by the procedures which are based among others on algorithms of wavelet transformation. The prepared scale diagrams, clearly show (Fig.5) that when $\omega_{G}=1.2$ and $\omega_{G}=1.35$, the periodical vibrations are excited in the gear. A comparison of the values of the parameter $\omega_{G}$ with the bifurcation diagram (Fig.3b) confirms the above formulated conclusions. In case of the chaotic attractors, it was observed that the harmonic components localized in a similar range of parameter $\omega$ are subjected to excitation. However, the scale diagrams show that in the particular time moments, other frequencies had the dominant role. The obtained results of model investigations i.e.: Poincare's cross sections, time responses (Fig.4) and scale diagrams (Fig.5), confirm the different gear dynamic behavior which depends on the changes of the control parameter $\omega_{G}$. However, the Fourier spectrums, with the exception of three cases, had a similar structure in the zone localized under the frequency $\omega_{G}$. Similarity of Fourier spectrums inspired us to make an attempt to identify initial attractors. From the theoretical point of view, points observed on Poincare's cross section arise when the trajectory passes through the control surface, whereas the surface could be chosen in an arbitrary manner. Usually, it is identical with the phase plane, which corresponds to angle $\alpha=2 \pi$. In the performed investigations, it was assumed that the angle defining the localization of the control surface could be a multiple of the angle $\alpha$. Effects of these investigations are shown graphically in Fig.6, where parameter $l_{m}$ defines multiplication of angle $2 \pi$. Based on the drawing of initial attractors, it is possible to formulate final conclusions:

- the dynamics of a nonlinear model of the considered gear, within the analyzed range of variability of parameters, corresponds to the same attractor;

- with an increase of the frequency $\omega_{G}$, the ends of ,arms” of an attractor are subjected to stretching or bending;

- parameter $l_{m}$ defining multiplication of angle $2 \pi$, is equal to the number of ,small tongues or flames" of the particular attractor, drawn for a control plane related to angle $2 \pi$,

- inaccuracies in manufacturing of gear parts after assembly of the whole unit can cause inaccuracies of transmission abilities of a particular gear.

Moreover, such analyses should be performed preventing of activity of the system in chaotic manner. The system should act in (safety) blue areas in Fig.3. So, designers have to carry out frequency analyses and pay more attention to some geometrical and related parameters like clearances, recurrence and uniformity.

\section{Nomenclature}

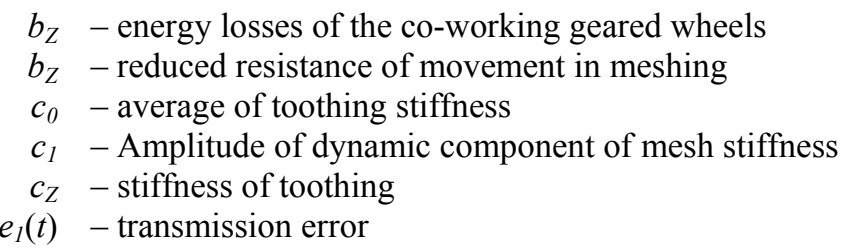




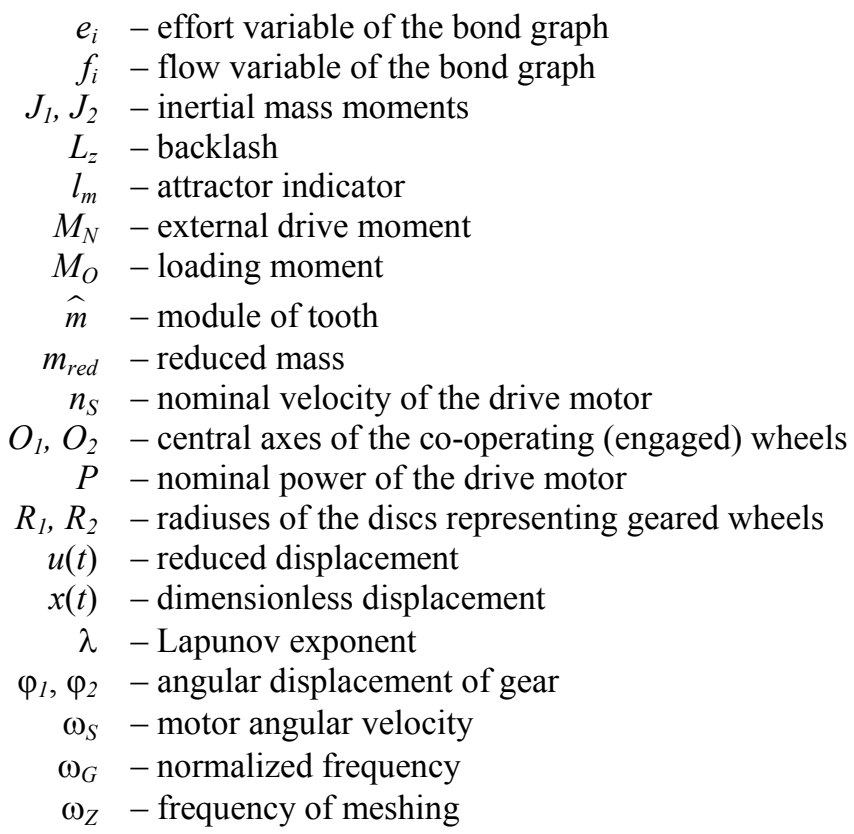

\section{References}

[1] Lorenz E.N. (1963): Deterministic non-periodic flow. - Journal of the Atmospheric Sciences, vol.20, pp.130-141.

[2] Cai-Wan Chang-Jian and Shiuh-Ming Chang (2011): Bifurcation and chaos analysis of spur gear pair with and without nonlinear suspension. - Nonlinear Analysis: Real World Applications, vol.12, pp.979-989.

[3] Khomeriki G. (2016): Parametric resonance induced chaos in magnetic damped driven pendulum. - Physic Letters A, 380, pp.2382-2385.

[4] Awrejcewicz J., Krysko-jr V.A., Pakovleva T.V. and Krysko V.A. (2017): Alternating chaos versus synchronized vibrations of interacting plane with beams. - International Journal of Non-Linear Mechanics, vol.88, pp.21-30.

[5] Armand Eyebe Fouda J.S., Bodo B., Djeufa G.M.D. and Sabat S.L. (2016): Experimental chaos detection in the Duffung oscillator. - Communications in Nonlinear Science and Numerical Simulations, vol.33, pp.259-269.

[6] Sajid I., Xizhe Z., Yanhe Z. and Jie Z. (2014): Bifurcation and chaos in passive dynamic walking, A review. Robotics and Autonomous System, vol.62, pp.889-909.

[7] Dudkowski D., Jafari S., Kapitaniak T., Kuznetsov N., Leonov G.A. and Prased A. (2016): Hidden attractors in dynamical system. - Physics Reports, vol.637, pp.1-50.

[8] Awrejcewicz J., Krysko A.V., Papkova I.V. and Krysko V.A. (2012): Routes to chaos in continuous mechanica systems. Part 3: The Lyapunov exponents, hyper-hyper and spatial-temporal chaos. - Chaos, Solitons and Fractals, vol.45, pp.721-736.

[9] Cai-Wan Chang-Jian (2012): Bifurcation and chaos analysis of the porous squeeze film damper mounted gearbearing system. - Computer and Mathematics with Application, vol.64, pp.798-812.

[10] Zhao Xin, Chen Changzheng, Liu Jie and Zhang Lei (2015): Dynamics characteristics of a spur gear transmission system for a wind turbine. - International Conference on Automation, Mechanical Control and Computational Engineering, pp.1985-1990.

[11] Kokare D.K. and Patil S.S. (2014): Numerical analysis of vibration in mesh stiffness for spur gear pair with method of phasing. - International Journal of Current Engineering and Technology, Special Issue 3, pp.156-159.

[12] Saghafi A. and Farshidianfar A. (2016): An analytical of controlling chaotic dynamics in a spur gear system. Mechanism and Machine Theory, vol.96, pp.179-191. 
[13] Cai Y. (1995): Simulation on the Rotational Vibration of Helical Gears in Consideration of the Tooth Separation Phenomenon (A New Stiffness Function of Helical Involute Tooth Pair). - "Journal of Mechanical Design", Transactions of the ASME, vol.117, pp.460-469.

[14] Cai Y. and Hayashi T. (1994): The Linear Approximated Equation of Vibration of a Pair of Spur Gears (Theory and Experiment). - Journal of Mechanical Design, Transactions of the ASME, vol.116, pp.558-564.

[15] Wang J., Guo L. and Wang H. (2013): Analysis of bifurcation and nonlinear control for chaos in gear transmission system. - Research Journal of Applied Sciences, Engineering and Technology, vol.6, No.10, pp.1818-1824.

[16] Xiang L., Yi J. and Aijun H. (2016): Bifurcation and chaos analysis for multi-freedom gear-bearing system with time-varying stiffness. - Applied Mathematical Modelling, vol.40, pp.10506-10520.

[17] Litak G. and Friswell M.I. (2003): Vibration in gear system. - Chaos, Solution and Fractals, vol.16, pp.795-800.

[18] Łuczko J. (2008): Chaotic vibrations in gear mesh systems. - Journal of Theoretical and Applied Mechanics, vol.46, No.4, pp.879-896.

[19] Ghosh S.S. and Chakraborty G. (2016): On optimal tooth profile modification for reduction of vibration and noise in spur gear pairs. - Mechanism and Machine Theory, vol.105, pp.145-163.

[20] Wang J., Zheng J. and Yang A. (2012): An analytical study of bifurcation and chaos in a spur gear pair with sliding friction. - International Conference on Advances in Computational Modeling and Simulation, Procedia Engineering, vol.31, pp.563-570.

[21] Zhang Y., Meng Z. and Sun Y. (2016): Dynamic modeling and chaotic analysis of gear transmission system in a braiding machine with or without random perturbation. - Shock and Vibration, Hindawi Publishing Corporation, Volume 2016, Article ID 8457645, 12 pages.

[22] Lang S.Y.T. (2005): Graph-theoretical modeling of epicyclic gear system. - Mechanism and Machine Theory, vol.40, pp.511-529.

[23] Wojnarowski J., Kopeć J. and Zawiślak S. (2006): Gear and graphs. - Journal of Theoretical and Applied Mechanics, vol.44, No.1, pp.139-151.

[24] Drewniak J. and Zawiślak S. (2010): Linear-graph and contour-graph-based models of planetary gear. - Journal of Theoretical and Applied Mechanics, vol.48, No.2, pp.415-433.

[25] Luo Y. and Tam D. (2011): Dynamics Modeling of Planetary gear set considering meshing stiffness based on bond graph. - Procedia Engineering, vol.24, pp.850-855.

[26] Guo Y., Liu D., Yang S., Li X. and Chen J. (2016): Hydraulic-mechanical coupling modeling by bond graph for impact system of a high frequency rock drill drifter with sleeve distributor. - Automation in Construction, vol.63, pp.88-99.

[27] Al-Shyyab A. and Kahraman A. (2005): Non-linear dynamic analysis of multi-mesh gear train using multi-term harmonics balance method: sub-harmonic motion. - Journal of Sound and Vibration, vol.279, pp.417-451.

[28] Jingyue W., Lixin G. and Haotion W. (2013): Analysis of bifurcation and nonlinear control for chaos in gear transmission system. - Research Journal of Applied Sciences, Engineering and Technology, vol.6, No.10, pp.1818-1824.

[29] Litak, G. and Friswell M.I. (2005): Dynamics of a gear system with faults in meshing stiffness. - Nonlinear dynamics 41.4, pp.415-421.

[30] Shuang L., Jin-Jin W., Jin-Jie L. and Ya-Qian L. (2015): Nonlinear parametrically excited vibration and active control of gear pair system with time-varying characteristic. - Chines Physical Society, vol.214, No.10, 8 pages.

Received: May 9, 2018

Revised: September 10, 2018 